\title{
Aportaciones de la economía del comportamiento en política sanitaria: Algunas notas en torno al ejemplo de la obesidad
}

\author{
ANNA GARCÍA-ALTÉS \\ Agència de Qualitat i Avaluació Sanitàries, (FIISS), c/ Roc Boronat 81-95, $2^{a}$ planta, 08005 \\ Barcelona, España.E-mail: agarciaaltes@gencat.cat
}

\begin{abstract}
RESUMEN
Los economistas del comportamiento estudian los efectos de los factores sociales, cognitivos y emocionales en las decisiones económicas de los individuos y las instituciones, y sus consecuencias. El paternalismo libertario defiende que las entidades del sector público y privado orienten las elecciones de la gente hacia direcciones que mejoren su calidad de vida. A partir del conocimiento de cómo la gente toma decisiones y sus sesgos, se pueden diseñar estrategias para que se tomen las más saludables. En la prevención de la obesidad, algunas de las intervenciones basadas en la economía del comportamiento han mostrado ser eficaces. Ahora bien, intervenciones multipalanca y, sobretodo, medidas reguladoras siguen siendo las más efectivas.
\end{abstract}

Palabras clave: Economía del comportamiento, paternalismo libertario, obesidad, regulación.

\section{Contributions of Behavioral Economics in Health Policy: Some Notes Around the Example of Obesity}

\begin{abstract}
Behavioural economists study the effects of social, cognitive, emotional and economic factors on individuals and institutions decisions, and their consequences. Libertarian paternalism argues that private and public sector entities should guide people's choices in directions that will improve their quality of life. Based on the knowledge of how people make decisions and their biases, strategies can be designed to take the healthiest choices. In the prevention of obesity, some interventions based on behavioural economics have proven efficatious. However, multifaceted interventions and, above all, regulatory measures remain the most effective.
\end{abstract}

Keywords: Behavioural Economics, Libertarian Paternalism, Obesity, Regulation.

Clasificación JEL: D7

Artículo recibido en junio de 2013 y aceptado en agosto de 2013

Artículo disponible en versión electrónica en la página www.revista-eea.net, ref. ə-31218 


\section{EL PATERNALISMO LIBERTARIO}

Los economistas del comportamiento estudian los efectos de los factores sociales, cognitivos y emocionales en las decisiones económicas de los individuos y las instituciones, y sus consecuencias. El campo se basa fundamentalmente en principios psicológicos, para sugerir medios de motivar a los individuos y los grupos para cambiar su comportamiento.

El término "paternalismo libertario" fue acuñado en los años 80, si bien ha tenido un fuerte renacimiento a partir del trabajo desarrollado por Richard $\mathrm{H}$. Thaler y Cass R. Sustein, profesores de economía y derecho de la Universidad de Chicago, conocidos también por haber trabajado en la campaña electoral de Barack Obama (Thaler y Sustein, 2003). El principio básico es sencillo: los seres humanos no son el Homo Economicus descrito en los libros de economía (que con información perfecta y después de razonar todas las alternativas, toma la mejor de las decisiones posibles, de acuerdo con sus preferencias), sino que se equivocan frecuentemente y cargan con las consecuencias de sus decisiones por mucho tiempo. Sin embargo, un "pequeño empujón” puede ser todo lo que se necesite para tomar una mejor decisión.

El aspecto libertario radica en la insistencia de que, en general, la gente debería ser libre para hacer aquello que le apetezca, descartando alternativas no deseadas. En este sentido, las políticas diseñadas mantendrán o aumentarán la libertad de decisión. El aspecto paternalista radica en el derecho de los "arquitectos de la elección” (gobiernos, empresas) a tratar de influir en el comportamiento de la gente para que viva más años, y tenga una calidad de vida mejor y más saludable. Se defiende que las entidades del sector público y privado hagan esfuerzos conscientes para orientar las elecciones de la gente en direcciones que mejoren su calidad de vida.

La regla de oro es ofrecer toques de alerta (“empujones”) que muy probablemente ayudarán y no serán perjudiciales. Un toque de alerta es cualquier aspecto de la arquitectura de la elección que modifica el comportamiento de la gente de una manera previsible, sin prohibir ninguna opción ni cambiar de manera significativa sus incentivos económicos (Thaler y Sustein, 2012). Para que algo sea considerado un toque de alerta, la intervención ha de ser fácil y barata de evitar.

Las aplicaciones de este concepto en salud pública para promover hábitos alimentarios saludables son numerosas, por ejemplo haciendo que la opción por defecto sea la opción saludable: no poner el salero en la mesa de los restaurantes, dar un único azucarillo al tomar un café en un bar, poner la fruta más cerca de los clientes que los pasteles en los comedores colectivos tipo autoservicio, etc. Sus aplicaciones en otras áreas de la promoción de la salud también son comunes: escribir en la calzada "look right/left" como en las calles de Londres, descuentos en los gimnasios para los clientes frecuentadores, cuentas de ahorro 
con el dinero no utilizado en comprar tabaco, etc.

\section{LOS DOS SISTEMAS}

La economía convencional considera que las personas son racionales: tienen conocimiento de todas las opciones disponibles, tienen preferencias que pueden clasificarse según sus gustos, y siempre escogen la opción que más les gusta. Sin embargo, desde la psicología se ha demostrado que a menudo las personas son irracionales y también que son previsiblemente irracionales. Si el conocimiento de las personas es previsiblemente irracional, entonces puede ser relativamente fácil incorporarlo en las teorías económicas para hacer predicciones más precisas. La economía del comportamiento pretende comprender mejor el comportamiento previsiblemente irracional de las personas (Ariely, 2008).

De alguna manera, se podría decir que la capacidad cognitiva humana está impulsada por dos sistemas, que no existen en la realidad, pero ayudan a explicar cómo las personas toman decisiones y a predecirlas (Kahneman, 2012). El sistema 1 funciona de manera automática y rápida, con poco o ningún esfuerzo y sin sentimiento de control voluntario; este sistema realiza operaciones automáticas. Generalmente, la capacidad cognitiva de las personas viene impulsada por este sistema de manera bastante eficiente. El sistema 2 dedica la atención a actividades mentales que requieren esfuerzo, cuando las decisiones o acciones son más complejas y requieren concentración, y es mucho más lento.

El sistema 1 se ejecuta de manera automática, sin esfuerzo; el sistema 2 se encuentra en modo de bajo esfuerzo, y sólo se utiliza una pequeña parte de él. El sistema 1 genera impresiones, intuiciones, sentimientos, intenciones; el sistema 2 las acepta. Cuando el sistema 1 se encuentra con una dificultad (cuando detecta un problema difícil o una cosa inesperada o improbable), pide al sistema 2 que le ayude. El sistema 1 no puede pararse, funciona con heurísticas determinadas y tiene sesgos. El comportamiento que de él se derive puede no ser racional, y el sistema 2 no necesariamente se dará cuenta. Una de las principales características del sistema 2 es la pereza, el rechazo a invertir más esfuerzo del que es estrictamente necesario. Mientras no se detecte nada extraño, la persona se limita a seguir las impresiones del sistema 1, sus intuiciones, sentimientos, etc.

El alejamiento de la racionalidad surge de los atajos (heurísticas) que las personas toman para valorar opciones complicadas. Estas heurísticas disminuyen la sobrecarga cognitiva, simplifican los problemas, pero conllevan errores en la toma de decisiones. Los científicos del comportamiento han identificado docenas de heurísticas y sus sesgos cognitivos relacionados, tales como los derivados de la emoción, las ideas preconcebidas, la disponibilidad de la información, las tendencias a la acción u omisión, y aversiones de varias clases (Tabla 1). El uso de tales controladores automáticos puede alejar a la gente de sus deseos 
conscientes y conducir a decisiones imperfectas y costosas. Algunos ejemplos en el sector sanitario pueden ser:

- El sesgo a tomar decisiones inmediatas de manera afectiva, hace recomendable no tomar las decisiones de costes en "caliente", ya que se descuidan objetivos a largo plazo.

- En negociaciones de precios, los costes de los servicios de salud pueden estar determinados por los precios pasados (ancla).

- Ante la tendencia a la acción, un médico puede elegir un tratamiento más agresivo frente a uno más conservador.

- El prejuicio del statu quo y la aversión a la pérdida hace que la ciudadanía pueda no estar dispuesta a renunciar a algunos servicios sanitarios, incluso cuando existen mejores alternativas.

Tabla 1

Heurísticas y sesgos en la toma de decisiones

\begin{tabular}{|l|l|}
\hline \multicolumn{1}{|c|}{ Sesgo } & \multicolumn{1}{c|}{ Significado } \\
\hline Afecto & $\begin{array}{l}\text { Las respuestas inmediatas suelen ser afectivas, orientando fuertemente el } \\
\text { procesamiento de la información y juicio posteriores }\end{array}$ \\
\hline Anclaje & $\begin{array}{l}\text { Tendencia a confiar en la primera pieza de información que se ofrece (ancla) } \\
\text { para tomar decisiones, aunque sea irrelevante }\end{array}$ \\
\hline Aversión a la pérdida & $\begin{array}{l}\text { Tendencia de las personas a preferir fuertemente evitar las pérdidas a tener } \\
\text { ganancias del mismo valor }\end{array}$ \\
\hline Acción & $\begin{array}{l}\text { Tendencia a favorecer la acción sobre la inacción, incluso cuando no hay un } \\
\text { objetivo en hacerlo }\end{array}$ \\
\hline Confirmación & $\begin{array}{l}\text { Tendencia a buscar o interpretar la información de una manera que confirme } \\
\text { las propias preconcepciones }\end{array}$ \\
\hline Descuento hiperbólico & $\begin{array}{l}\text { Tendencia a tener una preferencia fuerte por los beneficios inmediatos en } \\
\text { comparación con los beneficios futuros }\end{array}$ \\
\hline Disponibilidad & Tendencia a centrarse en el beneficio o suceso más destacado y más familiar \\
\hline Efecto foco & Tendencia a dar demasiada importancia a un aspecto particular \\
\hline Efecto marco & $\begin{array}{l}\text { Las personas se comportan de manera diferente en función de si una elección } \\
\text { se presenta como una ganancia o una pérdida }\end{array}$ \\
\hline Exceso de confianza & $\begin{array}{l}\text { Tendencia a tener exceso de confianza en el propio razonamiento intuitivo y } \\
\text { las capacidades cognitivas }\end{array}$ \\
\hline Identidad social & $\begin{array}{l}\text { Tendencia a anteponer la importancia de la pertenencia a un grupo a } \\
\text { argumentos sólidos }\end{array}$ \\
\hline Ilusión de control & $\begin{array}{l}\text { Tendencia a creer que se puede controlar, o al menos influir, en las } \\
\text { consecuencias o resultados que claramente no se pueden ni controlar ni influir }\end{array}$ \\
\hline Statu quo & Tendencia a valorar o apreciar en mayor medida las cosas estables \\
\hline
\end{tabular}

Fuente: Adaptado de King et al., 2013.

Ahora bien, la existencia de dos sistemas de procesamiento diferentes sugiere también la existencia de dos maneras diferentes de cambiar el comportamiento: modificando los procesos más automáticos de juicio (sistema 1), o modificando lo que la gente piensa de manera conscientemente (sistema 2) 
(King et al., 2013).

A pesar de la evidencia que demuestra la importancia del procesamiento automático en la toma de decisiones, la mayoría de instrumentos de política sanitaria -y no sanitaria- tradicionales se han centrado en el sistema 2. Vale la pena señalar que la teoría de la elección racional y muchos de los instrumentos de política que se derivan de ella, no es que sean erróneos cuando se refieren a los procesos de toma de decisiones, sino que son incompletos ya que ignoran los procesos automáticos. Las políticas basadas en la economía del comportamiento intentan dar cuenta de esta omisión modificando la arquitectura de la elección de dos maneras. En primer lugar, tratan de minimizar la toma de decisiones en el marco del sistema automático mediante el uso de estrategias que intentan alejar los decisores de los sesgos. Y, en segundo lugar, una vez reconocido que una parte de procesamiento automático no puede evitarse, intentar que los sesgos ayuden a tomar las decisiones correctas (King et al., 2013).

La arquitectura de la elección es el contexto en el cual se presentan las decisiones. Si los gobiernos o las organizaciones conocen como la gente toma decisiones y sus sesgos, pueden diseñar estrategias para que se tomen las más saludables. El comportamiento puede modificarse cambiando la "arquitectura de la elección” (Thaler y Sustein, 2012). El "mindspace framework" describe muchos de los elementos que afectan al comportamiento, sobre todo a través del sistema automático (sistema 1), y es útil para diseñar políticas que tengan por objetivo cambiar el comportamiento (Dolan et al., 2012) (Tabla 2).

Tabla 2

"Mindspace"

\begin{tabular}{|c|l|l|}
\hline M & Mensajero & Las personas están fuertemente influidas por quien comunica la información \\
\hline I & Incentivos & $\begin{array}{l}\text { Las respuestas de las personas a los incentivos están moduladas por atajos } \\
\text { mentales predecibles }\end{array}$ \\
\hline N & Normas & Las personas están fuertemente influidas por lo que hacen otras \\
\hline D & Defecto & Las personas siguen la opciones por defecto \\
\hline S & Señuelo & Los actos están a menudo influidos por señales subconscientes \\
\hline P & Prominencia & Las personas dirigen la atención a lo que es novedoso y parece relevante \\
\hline A & Afecto & Las asociaciones emocionales modifican las acciones de las personas \\
\hline C & Compromiso & $\begin{array}{l}\text { Las personas buscan ser consistentes con sus promesas públicas, y actuar en } \\
\text { consecuencia }\end{array}$ \\
\hline E & Ego & Las personas actúan de manera que se sientan bien con si mismas \\
\hline
\end{tabular}

Fuente: Adaptado de Dolan et al., 2012.

\section{LA OBESIDAD COMO EJEMPLO}

Existen algunos ejemplos de la aplicación de los conceptos de la economía del comportamiento a la prevención de la obesidad. La aplicación de la econo- 
mía estándar no ha sido demasiado exitosa en esta área, ya que no tiene en cuenta el pensamiento a nivel de grupo, la toma de decisiones en condiciones de incertidumbre y los procesos de toma de decisiones no racionales (Sobal y Bisogni, 2009; Capacci et al., 2012).

Las aplicaciones de la economía del comportamiento a las intervenciones dietéticas y la prevención de la obesidad incorporan estrategias heurísticas a los envases, precios, y promociones, necesarias para contrarrestar el uso de estas mismas estrategias por parte de la industria alimentaria. Los valores predeterminados son un método central para facilitar los comportamientos deseados, dada la tendencia de los individuos a mantener el statu quo (Johnson y Goldstein, 2003; Kahneman et al., 1991, Samuelson y Zeckhauser, 1988), tal y como se ha visto en los programas de donación de órganos y en el diseño de planes de jubilación (Thaler y Sunstein 2009). Otros métodos son los precios relativos de la comida más sana y menos sana, o las etiquetas en los menús para marcar la comida sana.

Diversos estudios de economía del comportamiento realizados en condiciones reales han demostrado ser efectivos para cambiar los patrones de compra de alimentos. Un ejemplo es una intervención dirigida a estudiantes de secundaria, en la que se requería el pago en efectivo de postres y refrescos (Just et al., 2007). Sin la opción de postres o refrescos, el pago podía realizarse mediante el cargo a tarjetas de crédito o débito. La aversión a perder dinero en efectivo para la compra de alimentos discrecionales redujo esas compras. Además, el cambio de comportamiento no disminuyó los ingresos de la cafetería (se ofrecía fruta y bebidas no edulcoradas por el mismo precio) ni el número de clientes, pero generó los efectos deseados de aumento de las ventas de alimentos más nutritivos y de reducción de las ventas de los menos nutritivos (Just y Wansink, 2009). Otro estudio realizado en el comedor de una escuela consistió en poner en marcha una "cola rápida" para pagar sólo los alimentos saludables, lo que hizo aumentar las ventas de estos alimentos en un 18\% (Hanks et al., 2012).

Desde hace poco tiempo, en Estados Unidos se está requiriendo a las cadenas de restaurantes que indiquen en los menús el número de calorías de los platos, a pesar de que la evidencia sobre la efectividad de esta intervención está en entredicho (Loewestein, 2011; Elbel et al., 2009). En cambio, sí existe evidencia sobre la influencia del tamaño del plato en la cantidad de comida que se come (Wansink et al., 2005), y sobre el impacto de los anuncios de comida mientras se está comiendo (Harris et al., 2010).

Sin embargo, la efectividad de las estrategias de economía del comportamiento a largo plazo es menos clara, y se desconoce si los comportamientos persisten en el tiempo o si surgen adaptaciones. Otro tipo de estrategias, como el uso de incentivos externos como factores de motivación para bajar de peso, han producido resultados mixtos (Halpern et al., 2012). 


\section{DISCUSIÓN}

En menos de una década desde que apareció el primer artículo sobre la aplicación de la economía del comportamiento, la idea ha sido bien acogida tanto entre los académicos, como en la industria y los gobiernos. A parte de la conocida participación en la campaña electoral de Barack Obama, en el Reino Unido el gobierno ha puesto en marcha un Behavioral Insights Team para apoyar la introducción de ideas de la economía del comportamiento en sus políticas (Cabinet Office, 2010). Sus principales atractivos recaen en las soluciones poco intrusivas y de bajo coste que ofrecen a algunos problemas relevantes para la sociedad actual, como el nivel bajo de ahorro o el consumo excesivo de energía.

Sin embargo, esta aproximación no está exenta de críticas (Marteau et al., 2011; Bonell, 2011). La principal sería que ninguna de estas ideas es nueva, y llevan mucho tiempo utilizándose en salud pública. Los supermercados y las marcas comerciales invierten cantidades ingentes de dinero en investigar cómo los consumidores toman decisiones según estén situados los productos. Las normas sociales y los incentivos son fundamentales en las teorías del cambio de comportamiento en salud, disponen de un marco teórico sólido, y se han usado innumerables veces. El marketing social usa constantemente estos mismos instrumentos. Los más críticos ponen de manifiesto que esta aproximación está siendo especialmente atractiva para gobiernos y países conservadores, en los que la intervención habitual del Estado en la vida diaria es mal recibida (Loewestein, 2012). Ahora bien, las opiniones más liberales no aceptan la intervención del Estado, ni en forma de estrategias de economía del comportamiento (Marlow y Abdukadirov, 2012).

En la prevención de la obesidad algunas de las intervenciones basadas en la economía del comportamiento han mostrado ser efectivas, al menos en el corto plazo. Ahora bien, intervenciones multipalanca que modifiquen el entorno alimentario, proporcionen educación nutricional, y empleen estrategias de la economía del comportamiento pueden contribuir potencialmente a una alimentación más saludable (Gittelsohn y Lee, 2013).

Con todo, una de las medidas más efectivas se halla en la política industrial, obligando a la industria alimentaria a que disminuya los contenidos de sal, azúcar y grasas en sus productos (NICE, 2010). La experiencia positiva de la ciudad de Nueva York con la regulación de las grasas “trans” puede ser un ejemplo a seguir. Los cambios de precios, la regulación de las etiquetas de los alimentos, y los límites al marketing dirigido a la población infantil están también entre las intervenciones más efectivas (Cecchini et al., 2010; Capacci et al., 2012). Las medidas impositivas son otra alternativa: si bien los impuestos sobre los productos con grasas y sal deberían ser descartados por su importante regresividad fiscal (Chouinard et al., 2007; Leicester y Windmeijer, 2005), los impuestos sobre las bebidas excesivamente azucaradas si podrían ser óptimos dada la poca 
elasticidad de la demanda de estos productos (López i Casasnovas, 2013). Sin embargo, los estudios no son conclusivos sobre los efectos de los impuestos en la prevalencia de obesidad en la población ni en su capacidad recaudatoria (Brownell et al., 2009; Lin et al., 2011).

\section{REFERENCIAS BIBLIOGRÁFICAS}

ARIELY, D. (2008). Predictably irrational: the hidden forces that shape our decisiones. New York HaperCollins.

BONELL, C.; MCKEE, M.; FLETHER, A.; WILKINSON, P.; HAINES, A. (2011) One nudge forward, two steps back: Why nudging might make for muddled public health and wasted resources. British Medical Journal, 342:241-242.

BROWNELL, FARLEY. The public health and economic benefits of taxing sugar-sweetened beverages. New England Journal of Medicine 361; 16:1599-1605.

CABINET OFFICE BEHAVIOURAL INSIGHTS TEAM. (2010). Applying behavioural insight to health. London: Cabinet Office Behavioural Insights Team.

CAPACCI, S.; MAZZOCCHI, M.; SHANKAR, B.; MACIAS, J.B.; VERBEKE, W.; PÉREZ-CUETO, F.J.; KOZIOŁ-KOZAKOWSKA, A.; PIÓRECKA, B.; NIEDZWIEDZKA, B.; D'ADDESA, D.; SABA, A.; TURRINI, A.; ASCHEMANNWITZEL, J.; BECH-LARSEN, T.; STRAND, M.; SMILLIE, L.; WILLS, J.; TRAILL, W.B. (2012). Policies to promote healthy eating in Europe: a structured review of policies and their effectiveness. Nutrition Reviews, 70(3):188-200.

CECCHINI, M.; SASSI, F.; LAUER, J.A.; LEE, Y.Y.; GUAJARDO-BARRON, V.; CHISHOLM, D. (2010). Tackling of unhealthy diets, physical inactivity, and obesity: health effects and cost-effectiveness. The Lancet, 376(9754):17751784.

CHOUINARD, H.H.; DAVIOS, D.E.; LAFRANCE, J.T.; PERLOFF, J.M. (2007) Fat taxes: big money for small change? Forum for Health Economics \& Policy, 10(2), pp. 1-28.

DOLAN, P.; HALLSWORTH, D.; HALPERN, D.; KING, D.; VLAEV, I. (2012). Influencing behaviour: the mindspace way. Journal of Economic Psychology;33(1):264-277.

ELBEL, B.; KERSH, R.; BRESCOLL, V.L.; DIXON, L.B. (2009) Calorie labelling and food choices: a first look at the effect on low-income people in New York City. Health Affairs (Millwood), 28, pp. 1110-1121.

GNEEZY, U.; MEIER, S.; REY-BIEL, P. (2011) When and Why Incentives (Don't) Work to Modify Behavior. Journal of Economic Perspectives, 25(4), pp. 191-210. 
GITTEELSOHN, J.; LEE, K. (2013) Integrating Educational, Environmental, and Behavioral Economic Strategies May Improve the Effectiveness of Obesity Interventions. Applied Economic Perspectives and Policy, 35 (1), pp. 52-68.

HALPERN, S.D.; ASCH, D.A.; VOLPP, K.G. (2012). Commitment Contracts As a Way to Health. British Medical Journal (Clinical research ed.), 344:e522.

HANKS, A.S.; JUST, D.R.; SMITH, L.E.; WANSINK, B. (2012). Healthy Convenience: Nudging Students Toward Healthier Choices in the Lunchroom. Journal of Public Health (Oxford, England, 34(3), pp. 370-376.

HARRIS, J.E.; BARGH, J.; BROWNELL, K. (2010). Priming effects of television food advertising on eating behavior. Health Psychology, 28(4), pp. 404-413.

JOHNSON, E.J.; GOLDSTEIN, D. (2003). Medicine. Do Defaults Save Lives? Science, 302(5649), pp. 1338-1339.

JUST, D.R.; PAYNE, C. (2009) Obesity: Can Behavioral Economics Help? Annals of Behavioral Medicine, 38(0), pp. 47-55.

JUST, D.R.; WANSINK, B. (2009) Better School Meals on a Budget: Using Behavioral Economics and Food Psychology to Improve Meal Selection. Choices, 24(3), pp. 19-24.

KAHNEMAN, D. (2012). Thinking fast and slow. Londres: Penguin Books.

KAHNEMAN, D.; KNETSCH, J.L.; THALER, R.H. (1991). Anomalies: The Endowment Effect, Loss Aversion, and Status Quo Bias. The Journal of Economic Perspectives, 5(1), pp. 193-206.

KING, D.; GREAVES, F.; VLAEV, I.; DARZI, A. (2013) Approaches Based On Behavioral Economics Could Help Nudge Patients And Providers Toward Lower Health Spending Growth. Health Affairs (Millwood), 32(4), pp. 661668.

LEICESTER, A.; WINDMEIJER, F. (2005). The 'fat tax': Economics incentives to reduce obesity. Institute for Fiscal Studies. Briefing Note num. 49, pp. 119.

LIN, B.H.; SMITH, T.A.; LEE, J.Y.; HALL, K.D. (2011). Measuring weigh outcomes for obesity interventions strategies: the case of a sugar-sweetened beverage tax. Economics and Human Biology, 9(4):329-341.

LOEWENSTEIN, G.; ASCH, D.A.; FRIEDMAN, J.Y.; MELICHAR, L.A.; VOLPP, K.G. (2012). Can behavioural economics make us healthier? British Medical Journal, 344:e3482.

LOEWESTEIN, G. Confronting reality: pitfalls of calorie posting. (2011). The American Journal of Clinical Nutrition, 93, pp. 679-680.

LÓPEZ I CASASNOVAS, G. (2013). El impuesto sobre bebidas excesivamente azucaradas. Barcelona: La Vanguardia, 13 MARZO.

MARLOW, M.L; ABDUKADIROV, S. (2012). Can Behavioural Economics Combat Obesity? Regulation, Summer 2012, pp. 14-18.

MARTEAU, T.M.; OGILVIE, D.; ROLAND, M.; SUHRCKE, M. (2011). Judging nudging: can nudging improve population health? British Medical Journal, 
342, pp. 263-265.

NICE. (2010). Prevention of cardiovascular disease at the population level. Public health guidance, PH25 - Issued: June 2010. London: NICE.

SAMUELSON, W.; ZECKHAUSER, R. (1988). Status Quo Bias in Decision Making. Journal of Risk and Uncertainty, 1, pp. 7-59.

SOBAL, J.; BISOGNI, C.A. (2009). Constructing Food Choice Decisions. Annals of Behavioral Medicine, 38 (Suppl. 1), pp. S37-S46.

STOREY, D.; LEE, K.; BLAKE, C.; LEE, H.Y.; DEPASQUALE, N.; LEE, P. (2011). Social \& Behavior Change Interventions Landscaping Study: A Global Review. Report Prepared for the Family Health Division of The Bill \& Melinda Gates Foundation.

THALER, R.H.; SUSTEIN, C.R. (2003). Libertarian paternalism. American Econonomic Review, 93, pp. 175-179.

THALER, R.H.; SUNSTEIN, C.R. (2009) Nudge: Improving Decisions About Health, Wealth, and Happiness. New York: Penguin Group.

WANSINK, B.; CHENEY, M.M. (2005) Superbowls: serving bowl size and food consumption. Journal of the American Medical Association, 293(14), pp. $1727-1728$. 